
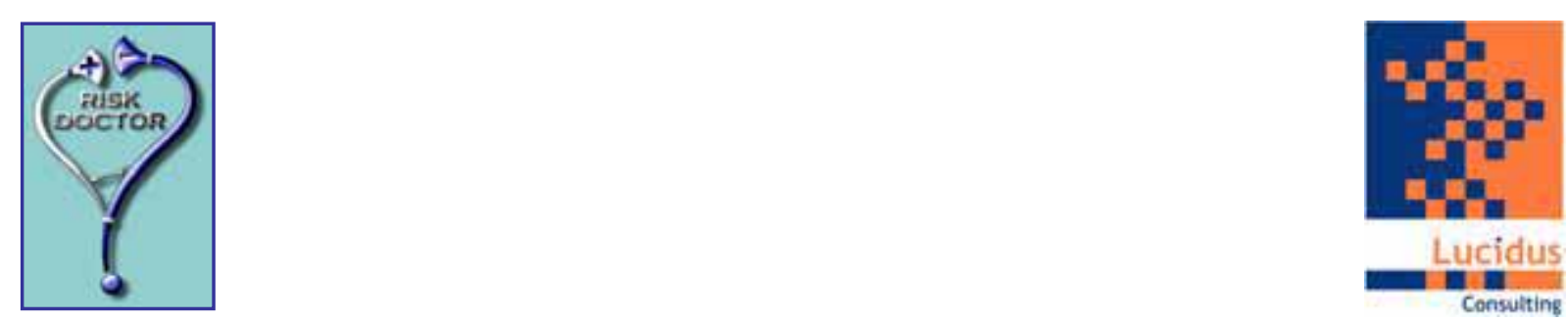

\title{
UNDERSTANDING AND MANAGING RISK ATTITUDE
}

\author{
David Hillson ${ }^{1}$ \& Ruth Murray-Webster ${ }^{2}$ \\ ${ }^{1}$ Director, Risk Do ct or \& Partner s, david@risk-doctor.com \\ ${ }^{2}$ Director, Lucidus Consulting, ruth@lucidusconsulting.com
}

\section{INTRODUCTION}

Risk management is recognised as an essential contributor to business and project success, since it focuses on addressing uncertainties in a proactive manner in order to minimise threats, maximise opportunities, and optimise achievement of objectives. There is wide convergence and international consensus on the necessary elements for a risk management process, and this is supported by a growing range of capable tools and techniques, an accepted body of knowledge, an academic and research base, and wide experience of practical implementation across many industries.

Despite this vision, in practice risk management often fails to meet expectations, as demonstrated by the continued history of business and project failures. Foreseeable threats materialise into problems and crises, and achievable opportunities are missed leading to lost benefits. Clearly the mere existence of accepted principles, well-defined processes, and widespread practice is not sufficient to guarantee success. Some other essential ingredient is missing.

The most significant Critical Success Factor for effective risk management is the one most often lacking : an appropriate and mature risk culture. Research and experience both indicate that the attitude of individuals and organisations has a significant influence on whether risk management delivers what it promises. Risk management is undertaken by people, acting individually and in various groups. The human element introduces an additional layer of complexity into the risk process, with a multitude of influences both explicit and covert. These act as sources of bias, creating preferred risk attitudes which affect every aspect of risk management. Risk attitudes exist at individual, group, corporate and national levels, and can be assessed and described with some degree of accuracy. This allows sources of bias to be diagnosed, exposing their influence on the risk process.

But diagnosis is different from cure. Where preferred risk attitude is not conducive to effective risk management, action is required to modify attitude. Recent advances in the field of Emotional Intelligence provide a means by which attitudinal change can be promoted and managed, for both individuals and organisations.

It is important firstly to understand risk attitudes and the impact they can have on the risk management process if their presence and influence are not recognised or managed. It is also important to understand how development of emotional intelligence can provide practical and powerful tools for modifying risk attitudes. 


\section{WHAT IS RISK?}

The word "risk" is a common and widely-used part of today's vocabulary, relating to personal circumstances (health, pensions, insurance, investments etc), society (terrorism, economic performance, food safety etc), and business (corporate governance, strategy, business continuity etc). Yet somewhat surprisingly, there is still no broad consensus on the meaning of this term. Various national and international standards and guidelines exist which mention risk, but there are many different definitions and underlying concepts in these documents. Even among risk practitioners in the various professional bodies there is an ongoing debate about the subject matter at the heart of their discipline. And of course there is huge variation in the general literature, reflecting the lack of official agreement on the basic definition of risk.

Despite differences of detail, all definitions agree that risk has two characteristics : it is related to uncertainty, and it has consequences. Risk however is not the same as uncertainty, whether aleatoric variability or epistemic ambiguity. The key distinction between uncertainty and risk arises from consideration of the consequences. Perhaps the simplest definition of risk is "Uncertainty that matters", since uncertainty without consequence poses no risk. In this sense, risk cannot be defined unless it is related to objectives of some kind. A more complete definition therefore might be "an uncertainty that could have a positive or negative effect on one or more objectives". This is illustrated in Figure 1, where risk arises from uncertainties that if they occurred might affect the typical project objectives of time/cost/scope.

Defining this link between risk and objectives is essential to the process of risk management, since it is a prerequisite for identifying risks, assessing their significance, and determining appropriate responses. It is also however a crucial factor in understanding risk attitudes, since these are driven by the objectives of the individual, group or organisation concerned, and the extent to which the risk "matters".

\section{Risk arises from interactions between objectives \& uncertainty}

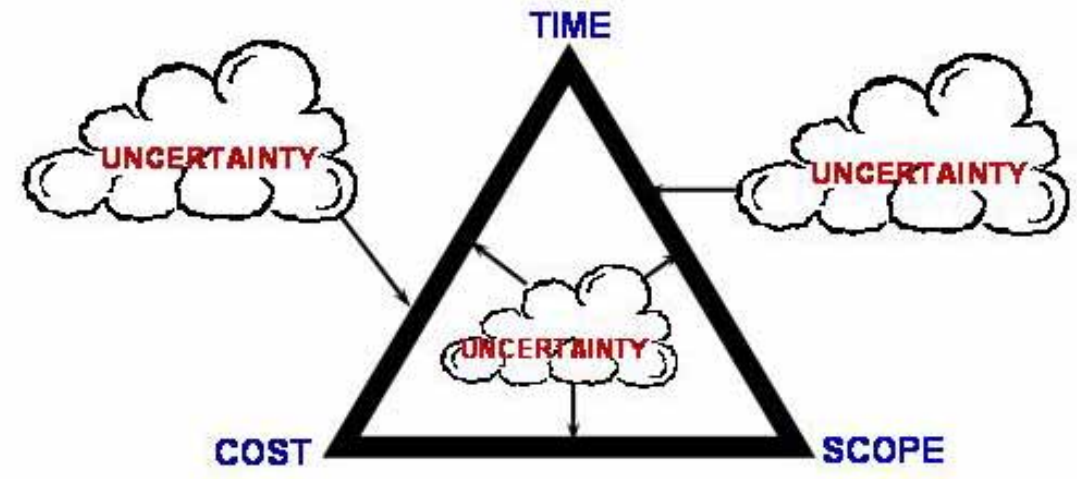

Figure 1: Risk, objectives \& uncertainty

\section{WHAT IS ATTITUDE?}

Attitude is another word used commonly but loosely. Dictionaries offer two differing definitions. The first relates to the inner working of the human mind, where "attitude" is "state of mind, mental view or disposition with regard to a fact or state". A second equally valid definition describes the positioning of an object in space, such as an aircraft, spaceship, or missile, where "attitude" is said to mean "orientation of axes in relation to some reference plane, usually the horizontal". 
It is interesting to note that both definitions insist that attitude can only exist in relation to a datum point - either a fact towards which one holds a mental disposition, or a reference plane such as the horizon against which orientation is measured. In this respect "attitude" is similar to "risk", which is defined in terms of objectives.

Although at first sight mental views and aircraft positioning do not seem to have much in common, in fact the two definitions of attitude are not incompatible or unrelated.

- Just as the pilot makes a decision on what attitude to adopt for the aircraft in threedimensional space in order to position it to execute the desired manoeuvre, so an individual or group can make an attitudinal choice to lean towards a particular desired response, behaviour or outcome.

- The attitude of an aircraft does not in itself result in motion, although it is a direct influence on the direction taken. In addition to attitude some force must act on the aircraft to generate motion - analogous to motivation.

- Aircraft attitude needs to be followed by movement if it is to result in execution of a manoeuvre, and similarly individual or group attitudes must be translated into action if the desired outcome is to be achieved.

- Attitude in space can be described using a number of elements, usually termed "pitch", "roll" and "yaw". It is also possible to subdivide human attitudes into their component dimensions to enable them to be better understood and managed.

- As the number of degrees of freedom for aircraft movement is almost unlimited within the three dimensions of space, so there is a bewildering array of potential attitudes that can be chosen in any given situation.

- It is possible for extremes of attitude to make an aircraft unstable (for example stall or spin), resulting in loss of control and potentially catastrophic consequences. Similarly a sense of balance is required for individuals and groups if their attitudes are not to lead to undesired outcomes.

- Different extremes of attitude require different types of response. For example if an aircraft finds itself in a stall the correct response is to do nothing, allowing the aircraft to self-correct. In the case of spin however emergency action is required to bring the aircraft under control. In the same way some extremes of human attitude are selfcorrecting where others require aggressive intervention.

- While there may be a preferred response (initial default positioning), the final outcome remains a matter of choice.

As a result of this comparison, the term "attitude" as applied to internal human mental processes and positioning is used here to refer to chosen responses to situations. Some attitudes may be deeply rooted, representing core values for the individual or group, but they nevertheless represent a choice. Other attitudes may be more malleable. Attitudes differ from personal characteristics in that they are situational responses rather than natural preferences or traits, and chosen attitudes may therefore differ depending on a range of different influences. Clearly if these influences can be identified and understood, the possibility of changing them is introduced, allowing individuals and groups to manage their attitudes proactively - which is the basis of emotional literacy.

This attribute of attitudes to be capable of modification is essential to the case for understanding and managing risk attitudes. If attitudes were fixed inherent attributes of individuals, inborn and unchangeable, then while it might be possible to understand them it would never be possible to manage them. The attitudes of individuals or groups would then not be comparable to an aircraft flying freely through the air, but would instead be like a cruise missile pre-programmed to strike a fixed target.

Page 3 of 11

(C) Copyright 2004 David Hillson/Ruth Murray-Webster 


\section{WHAT IS RISK ATTITUDE?}

If "risk" is defined as "an uncertainty that could have a positive or negative effect on one or more objectives", and "attitude" is defined as "chosen state of mind, mental view or disposition with regard to a fact or state", then combining the two gives a working definition of "risk attitude" as "chosen state of mind with regard to those uncertainties that could have a positive or negative effect on objectives", or more simply "chosen response to perception of significant uncertainty".

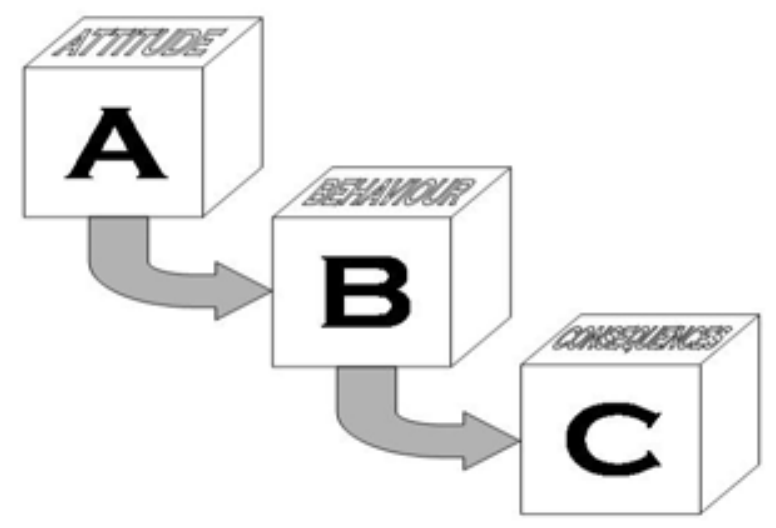

Figure 2: $A B C$ of risk psychology

A range of possible attitudes can be adopted towards the same situation, and these result in differing behaviours, which lead to consequences, both intended and unintended (see Figure 2). Indeed behaviour is the only reliable diagnostic indicator of inner attitude, and considerable attention has therefore been paid to behavioural psychology and management by those seeking to understand and manage the effects of human factors in business. Another approach however, which might prove more fruitful, is to seek to understand and address the underlying attitudes, rather than concentrating on the presenting behavioural symptoms.

Although attitude manifests itself through behaviour, there are other drivers of behaviour which can displace the chosen or preferred attitude. The extent to which this occurs depends on the perception of the situation towards which the attitude is being directed. This is best understood by considering the two extremes, where the situation is perceived as good or neutral, and where it is seen as bad.

- Favourable or neutral situation. When a situation or environment is perceived as positive or benign, behaviour is driven largely by attitude. In this case the attitudinal choice of the individual or group is the key determinant of behaviour. For example when faced with an existing client who is open to the possibility of taking on new business, an organisation may decide to pursue the opportunity or to ignore it ("take it or leave it"). This choice is not mandated by the situation, and the organisation is free to select its preferred response. People who adopt this attitude consistently may be labelled as optimists, since they tend to view all situations as equally positive. This helps them to retain control of their behaviour since the key driver when the environment is positive is the chosen attitude, allowing a proactive response to the prevailing situation. 
- Unfavourable or hostile situation. When an individual or group perceives a situation or environment as negative, the resulting behaviour is largely determined by a direct response to the situation, and attitude plays a smaller role. For example in a setting where one's personal safety is threatened, the "fight or flight or freeze" response is adopted almost unthinkingly, regardless of the prevailing attitude of the individual. Indeed a negative situation may force behaviour which is contrary to that preferred by attitude, leading to a more reactive stance. Individuals who regularly adopt reactive behaviour driven by a perception that the environment is negative may be termed pessimists, and in extreme cases this may even lead to paranoia.

Although the responses to positive and negative situations suggest at first sight that environment or situation is the prime determinant of behaviour, in fact it is how the environment is perceived by each person, since a situation that appears hostile to one may seem benign to another. This raises the question of what influences behaviour when the situation is uncertain. In this case the important driver of behaviour is whether uncertainty is perceived as favourable, neutral, unfavourable or hostile. This reaction to uncertainty is "risk attitude", defined above as "chosen response to perception of significant uncertainty".

One key conclusion on which researchers and practitioners are agreed is that risk attitudes exist on a spectrum, as shown in Figure 3. The same uncertain situation will elicit different preferred attitudes from different individuals or groups, depending on how they perceive the uncertainty. And since attitude drives behaviour, different people will exhibit different responses to the same situation, as a result of their differing underlying risk attitudes (sometimes called "perceptual dissonance") - a situation regarded as too risky by one person will be seen as acceptable by another.

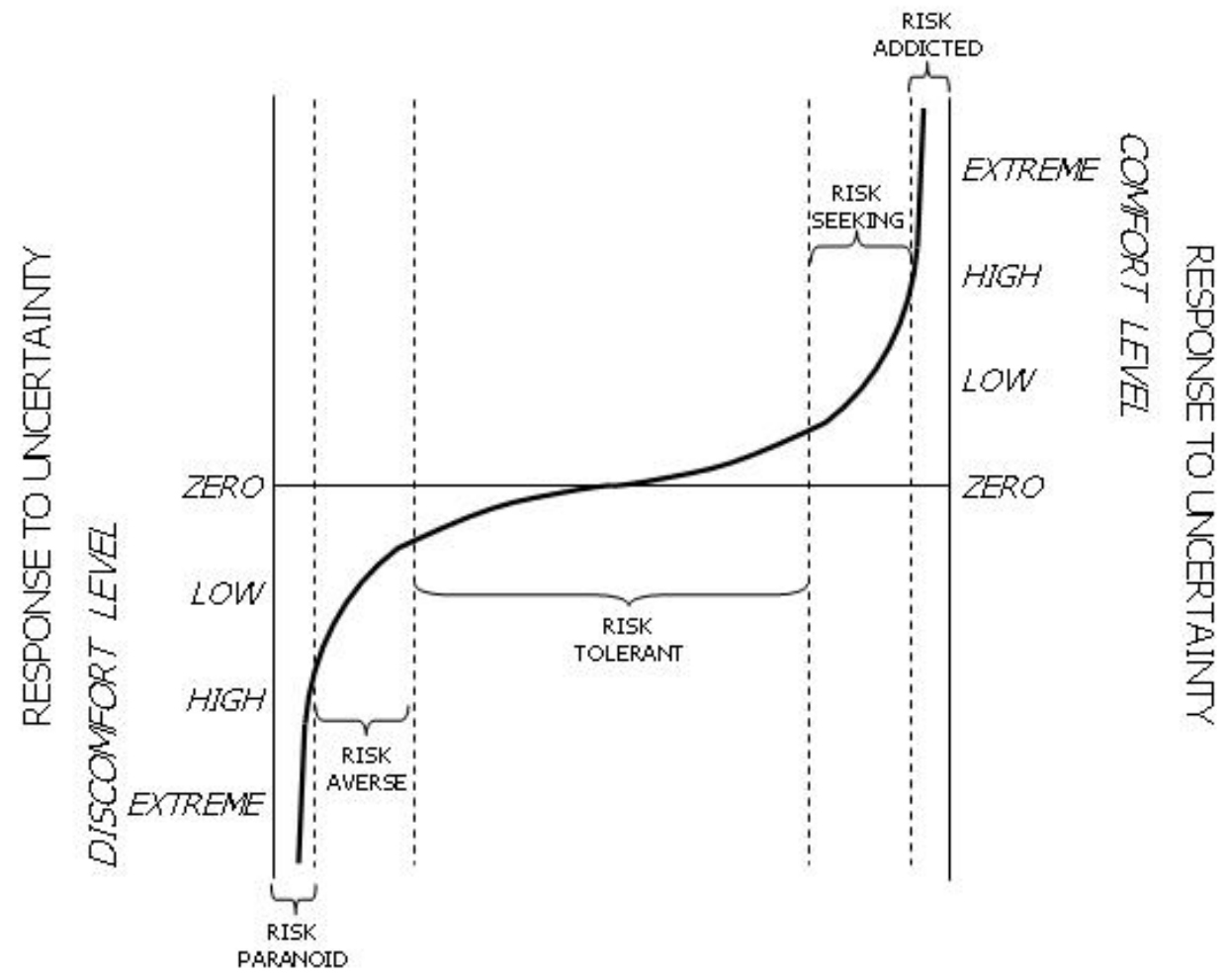

Figure 3 : Risk attitude spectrum 


\section{WHAT IS EMOTIONAL INTELLIGENCE}

Everyone experiences emotions throughout their waking and working day, and even when they sleep. Yet like the term "risk", there is no single widely-accepted definition. All standard dictionaries offer definitions for the term "emotion"; from "disturbance of mind", to "mental sensation or state". The fact that a particular person feels fear is only meaningful in context. If the fear is preventing the person from pursuing a course of action that would be beneficial for them, the emotion is negative. Conversely if the fear is preventing the person for pursuing a dangerous course of action then the emotion is positive. Emotions in themselves have no absolute meaning, their significance is only important in relation to the objectives that people seek to achieve.

However much people may like to believe that in work situations they behave logically, analysing problems and making decisions in a rational way, the reality is that emotions are always present, influencing behaviour and actions. The rich English vocabulary has many words to describe how people feel. Some emotions are so primal, e.g. fear, anger or desire that it is difficult for people to consciously over-ride their subconscious drive to "go with their feeling". Less emotive words may be used for these feelings, e.g. anxious, cross or excited, but the effect is the same. Resultant actions may be positive or negative, empowering or debilitating, but it is certain they will affect behaviour. Not only do emotions drive the actions of individuals, they also affect the wider groups in which people work, and vice versa.

Whilst the world would be a lesser place without spontaneity, the route to effective decisionmaking begins with individuals being cognisant of the emotions that are driving them in any particular situation. This awareness does not make the emotion go away, although awareness and understanding enables the choice to change, but it does provide the basis for harnessing emotions to produce results that lead towards rather than away from goals. Accordingly, it is asserted that "If you know you, you won't get in the way(... of your decisionmaking processes)", and this is directly relevant to decision-making in general, and effective risk management in particular.

This assertion leads to two central concepts :

- Emotions can be recognised, understood, appropriately expressed and managed

- People can harness emotions to help themselves and others succeed

Emotional Intelligence offers an approach towards achieving these two aims.

Although the term "emotional intelligence" has only been part of common parlance in the last decade, the concept, as with most things, is not new : philosophers and psychologists for centuries have been stressing the importance of understanding one's own emotional state. However, up until recent times, business attitudes in the West at least have tended to favour a view that "emotions are best kept private and under a tight rein". Personal power for too long has been linked to "nerves of steel" and the capacity to be emotionally detached and cool. These views have changed in more recent years and it is recognised that personal power depends on having a comfortable relationships with emotions. Emotional intelligence requires that emotions be listened to and expressed in a productive way.

In 1983, the psychologist Howard Gardner demonstrated that human beings display intelligence in a number of different ways, from the classically understood linguistic and logical-mathematical types of intelligence, through intra-personal and inter-personal intelligences to areas such as musical intelligence. The classic view of intelligence (measured by the Intelligence Quotient (IQ) and focused on the use of logical reasoning, rational analysis and spatial orientation to solve problems) was not the whole story. 
Accordingly when Salovey and Mayer in 1989 formally introduced the term "emotional intelligence", it made real sense to people who intuitively already knew that IQ was not the only determinant of success in a business context. Goleman popularised the term, and there is now a wealth of literature dedicated to helping individuals understand how they can become more emotionally intelligent and thus be more successful in attaining their goals. The key questions are "How does natural, intuitive behaviour affect actions?" and "How can individuals acknowledge it (at least) and continually and intentionally develop it (if they choose to)?"

Although there is no absolute agreement between authors on the features that together make an emotionally intelligent person, there is considerable overlap and enough synergy to draw conclusions about the critical dimensions and how they map to the four major stages required to develop emotional literacy, as shown in Table 1.

\begin{tabular}{|c|c|}
\hline $\begin{array}{l}\text { 1. RECOGNISE EMOTIONS } \\
\text { - Self-awareness } \\
\text { - } \quad \text { Ompathy } \\
\text { - } \quad \text { Truanst }\end{array}$ & $\begin{array}{l}\text { 2. UNDERSTAND EMOTIONS } \\
\text { - } \quad \text { Relative regard } \\
\text { - } \quad \text { Personal power and Self-confidence } \\
\text { Flexibility/Behavioural adaptability }\end{array}$ \\
\hline $\begin{array}{l}\text { 3. APPROPRIATELY EXPRESS EMOTIONS } \\
\text { - Goal directedness and Emotional self- } \\
\text { - } \text { control } \\
\text { - } \\
\text { - } \text { Assertivenenens and Conflict handling } \\
\text { - } \\
\text { Constructive discontent }\end{array}$ & $\begin{array}{l}\text { 4. MANAGE EMOTIONS } \\
\text { - Intentionality//Impulse control } \\
\text { - Interional resilience/Stress tolerance } \\
\text { - } \text { General health and Quality of life }\end{array}$ \\
\hline
\end{tabular}

$\underline{\text { Table } 1 \text { : Components of emotional literacy }}$

\section{EMOTIONAL INTELLIGENCE AND RISK MANAGEMENT}

It is inherent in the nature of risk management for it to be exposed to sources of explicit and implicit bias, since all elements of the risk process are performed by individuals and groups of people whose risk attitudes affect every aspect of risk management. Risk attitudes exist at individual and group levels, and these can be assessed and described with some degree of accuracy. Sources of bias can also be diagnosed, exposing their influence on the risk process.

Where the risk attitude adopted is not conducive to effective risk management, action is required to modify attitude. Emotional Intelligence provides a means by which attitudinal change can be promoted and managed, for both individuals and groups.

Risk has been defined as uncertainty that matters, including both uncertainties which pose a threat or uncertainties that present opportunities. In a similar way, Emotional Intelligence in the context of risk management is about emotions that matter. This recognises that emotions can assist people in managing risk, or they can be a hindrance. All elements of the risk process are affected by the Emotional Intelligence of the stakeholders, at both individual and group levels, including:

- Identification of risks

- Assessment of probability of the risk occurring

- Assessment of potential impact should the risk occur

- Deciding how to respond to assessed risks 
Decision-making is an activity that consumes a large percentage of time for many individuals and groups particularly in the workplace, and this is definitely the case for people involved in the discipline and profession of risk management. However decision-making in uncertain situations is affected by "risk attitude", influenced by numerous sources of explicit and implicit bias. One of most the significant contributors to risk attitude is the emotional state of the individual or group involved and therefore development of Emotional Intelligence will have a direct effect on the efficacy of the risk management process.

Risk attitudes are usually adopted sub-consciously. Free-flowing subconscious behaviour can be good in that it allows people to make good decisions in uncertain situations quickly and without effort. It can also be bad if the habitual behaviour is ineffective and continually results in sub-optimal decisions being made. Like any other attitude however, risk attitudes represent a choice. Some people may not immediately accept this, describing themselves or others as inherently risk averse, or risk seeking and this is most likely to be because the choice has become so deeply habitualised that the person behaves as is there is no choice. In contrast other individuals and groups have learned to assess each situation internally, and then to choose a risk attitude explicitly, selecting the attitude which is most appropriate to the situation and which offers the best chance of achieving their objectives.

Clearly the emotionally literate approach involves both awareness and action, which is likely to be more effective than "choosing not to choose" and simply adopting whatever risk attitude comes naturally.

Figure 4 presents a simple process where the application of Emotional Intelligence can be used to assess risk attitude and to modify it where necessary. Each step in this process can be implemented by answering a series of questions designed to encourage self-awareness and promote self-modification. The same process can be applied by either individuals or groups. [Full details of this process are available from the authors on request.]

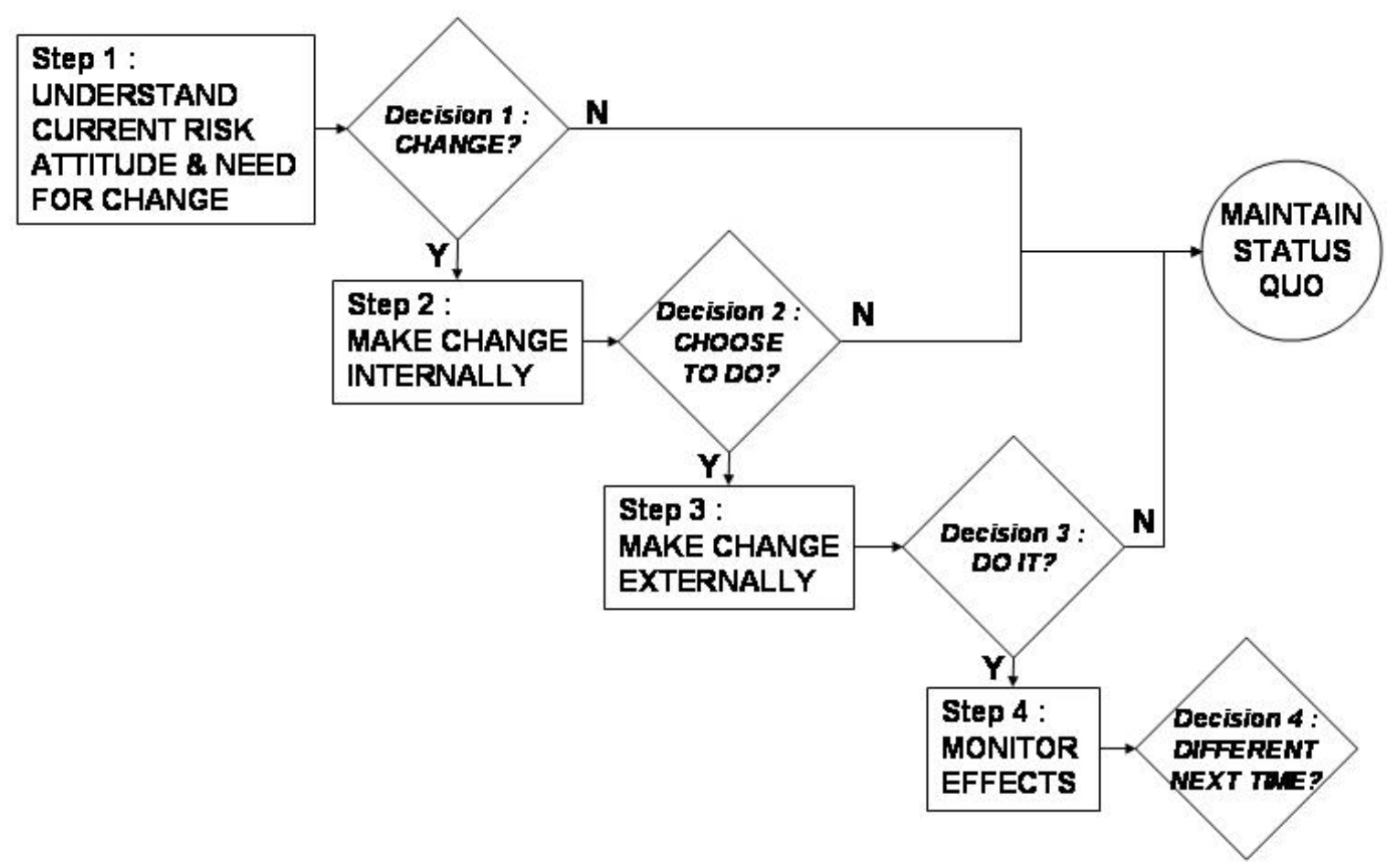

Figure 4 : Applying Emotional Intelligence to assess and modify risk attitude 


\section{CONCLUSION}

Risk matters. Human factors matter. Risk attitudes matter. Emotions matter.

Each of these statements is connected by a focus on achievement of objectives, which are the measure of "mattering". The various dimensions of risk, human factors, risk attitude and emotions can each be described and assessed in isolation using well-proven models and frameworks. But they interact in powerful ways, and those interactions play a significant part in determining the effectiveness of each separate part. For individuals, groups and organisations who need to ensure that management of risk is effective, it is essential that they understand and manage all elements of this complex web. What part do human factors play in the risk management process? How are risk attitudes adopted and modified? How does the perception of risk affect behaviour and decision-making? Why are emotions important in the workplace?

Despite the complexities of this challenge, some core concepts can be defined and the broad outlines of a solution exist. Applying the proven techniques of emotional literacy will start to unveil the mysteries of risk attitudes and allow steps to be taken towards improved risk management effectiveness.

Subconscious and unmanaged risk attitudes pose a significant threat to the ability of individuals and groups to achieve their objectives. Developing emotional literacy at both individual and group levels offers a route towards understanding and managing risk attitudes, allowing the undoubted benefits to be reaped and creating a framework for ongoing learning and increased risk management effectiveness.

\section{ACKNOWLEDGEMENT}

The material in this paper and the accompanying presentation is taken from the forthcoming book "Understanding and Managing Risk Attitude" by David Hillson and Ruth MurrayWebster, to be published by Gower Publishing Limited in 2005 (ISBN 0-566-08627-1). The concepts expressed in this material are the copyright of David Hillson and Ruth MurrayWebster and must not be reproduced or copied in whole or in part under any circumstances.

\section{ABOUT THE AUTHORS}

Dr David Hillson PMP FAPM FIRM is an international risk management consultant, and Director of Risk Doctor \& Partners (www.risk-doctor.com). He is a popular conference speaker and awardwinning author on risk. He is recognised internationally as a leading thinker and practitioner in the risk field, and has made several innovative contributions to improving risk management, most recently the inclusion of proactive opportunity management within the risk process. David is an active member of the Project Management Institute (PMI) and received the 2002 PMI Distinguished Contribution Award for his work in developing risk management over many years. He is a Fellow of the UK Association for Project Management (APM) and a Fellow of the UK Institute of Risk Management (IRM).

Ruth Murray-Webster PMP MAPM MBA is Director of Lucidus Consulting Limited (www.lucidusconsulting.com). Ruth has particular interests in programme management, quality management and the development of cultural fluency within project teams. This interest is supported by a wide experience of managing international projects and programmes in highly regulated industries. She is a highly-qualified and respected business consultant, with broad experience in managing organisational change in a cross-cultural context and across a wide range of sectors, and a particular interest in the human aspects of effective change management. 


\section{REFERENCES}

Association for Project Management. 2004 "Project Risk Analysis \& Management (PRAM) Guide" (second edition). Published by APM Publishing, High Wycombe, Bucks UK. ISBN 1-903494-12-5

BS IEC 62198:2001 "Project risk management - Application guidelines". Published by British Standards Institute, London, UK. ISBN 0-580-390195

BSI PD 6668:2000 "Managing risk for corporate governance". Published by British Standards Institute, London, UK. ISBN 0580-33246-2

Chernis C. \& Goleman D. (eds) 2001. "The emotionally intelligent workplace". Published by JosseyBass, San Francisco US. ISBN 0-7879-5690-2

Covey S. R. 1990. "Seven Habits Of Highly Effective People". Published by Free Press. ISBN 0671708635

Druskat V. U. \& Wolff S. B. 2001. "Building the Emotional Intelligence of groups". Harvard Business Review, Volume 79 Number 3, pages 80-90

Gardner H. 1993. "Frames of Mind: The Theory of Multiple Intelligences" (tenth anniversary edition). Published by Basic Books, New York, US. ISBN 0465025102

Gilovich T. Griffin D. \& Kahneman D. (eds). 2002. "Heuristics and biases : The psychology of intuitive judgement". Published by Cambridge University Press, Cambridge UK

Goleman D. 1995. "Emotional Intelligence : Why it can matter more than IQ". Published by Bloomsbury Publishing plc, London UK. ISBN 0-7475-2830-6

Goleman D. 1998. "Working with Emotional Intelligence". Published by Bloomsbury Publishing plc, London UK. ISBN 0-7475-3984-7

Goleman D. 2001. "Emotionally intelligent workplace : How to select for, measure and improve emotional intelligence in individuals, groups and organisations". Published by Jossey-Bass, Hackensack, NJ, US

Goleman D. 2003. "Destructive Emotions". Published by Bloomsbury Publishing plc, London UK. ISBN 0-7475-5393-9

Goleman D., Boyatzis R. \& McKee A. 2004. "Primal leadership: Learning to lead with Emotional Intelligence". Published by Harvard Business School Press. ISBN 1591391849

Hillson D. A. 2002 "Critical Success Factors for Effective Risk Management", David Hillson, PM Review, (four-part series) July-December 2002

Hillson D. A. 2002. "What is risk? Towards a common definition". InfoRM, journal of the UK Institute of Risk Management, April 2002, pages 11-12

Hillson D. A. 2003. "Effective opportunity management for projects : Exploiting positive risk." Published by Marcel Dekker, New York, US. ISBN 0-8247-4808-5

Hillson D. A. \& Murray-Webster R. 2004. "Understanding and managing risk attitude". Proceedings of $7^{\text {th }}$ Annual Risk Conference, held in London, UK, 26 November 2004.

Hillson D. A. \& Murray-Webster R. 2005. "Understanding and managing risk attitude using applied emotional literacy". Published by Gower, Aldershot, UK. ISBN 0-566-08627-1 (forthcoming)

Hofstede G. H. 2001. "Culture's consequences : Comparing values, behaviours, institutions, and organisations across nations" (second edition). Published by Sage Publications Inc, Thousand Oaks, California US. ISBN 0-8039-7324-1

Institute of Risk Management (IRM), National Forum for Risk Management in the Public Sector (ALARM), \& Association of Insurance and Risk Managers (AIRMIC). 2002. "A risk management standard". Published jointly by IRM/ALARM/AIRMIC, London UK 
Kahneman D. \& Tversky A. 1979. "Prospect theory : An analysis of decision under risk". Econometrica, Volume 47 Number 2, pages 263-297

Kahneman D., Slovic P. \& Tversky A. (eds.) 1986. "Judgement under uncertainty : Heuristics and biases". Published by Cambridge University Press, Cambridge, UK.

Lopes L. L. 1987. "Between hope and fear : The psychology of risk". Advances in Experimental Social Psychology, Volume 20, pages 255-295

Mayer J. D. \& Geher G. 1996. "Emotional intelligence and the identification of emotion". Intelligence, Volume 22, pages 89-113.

Project Management Institute. 2004. "A Guide to the Project Management Body of Knowledge $\left(\right.$ PMBoK $\left.^{\circledR}\right) "$, Third Edition. Project Management Institute, Philadelphia, US.

Salovey P., Brackett M. A. \& Mayer J. D. 2004. "Emotional Intelligence: Key Readings on the Mayer and Salovey Model". Published by National Professional Resources Inc. ISBN 1887943722

Schein E. H. 2004. "Organisational culture and leadership" (third edition). Published by Jossey-Bass. ISBN 07879-68455

Schneider S. L. \& Shanteau J. (eds). 2003. "Emerging perspectives on judgement and decision research". Published by Cambridge University Press, Cambridge UK

Slovic P. 2000. "Perception of risk". Published by Earthscan Press, London UK

Slovic P., Finucane M. L., Peters E. \& MacGregor D. G. 2004. "Risk as analysis and risk as feelings : Some thoughts about affect, reason, risk and rationality". Risk Analysis, Volume 24 Number 2, pages 311-322

Slovic P. 2005. "Emotion and reason in learning about risk". Proceedings of ESRC Social Contexts And Responses to Risk (SCARR) Launch Conference, held in Canterbury, UK, 28-29 January 2005.

Steiner C. \& Perry P. 2000. "Achieving Emotional Literacy" (second edition). Published by Barnes \& Noble. ISBN 0747541353

Trompenaars F. \& Hampden-Turner C. 1998. "Riding the waves of culture" (second edition). Published by McGraw-Hill. ISBN 0-7863-1125-8

Tversky A. \& Kahneman D. 1974. "Judgement under uncertainty : Heuristics and biases". Science, Volume 185, pages 1124-1131

Tversky A. \& Kahneman D. 1981. "The framing of decisions and the psychology of choice". Science, Volume 211, pages 453-458

UK Office of Government Commerce (OGC). 2002. "Management of Risk - Guidance for Practitioners". Published by The Stationery Office, London, UK. ISBN 0-1133-0909-0

Yates J. F. (ed) 1992. "Risk-taking behaviour". Published by J Wiley, Chichester, UK. ISBN 0-47192250-1 